\title{
A high-resolution, variable-energy electron beam from a Penning-Malmberg (Surko) buffer-gas trap
}

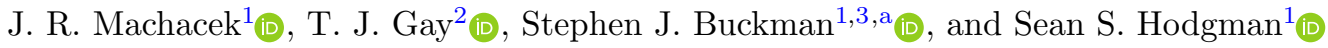 \\ 1 Research School of Physics, Australian National University, Canberra, ACT, Australia \\ 2 Department of Physics and Astronomy, University of Nebraska, Lincoln, NE 68588-0299, USA \\ 3 Department of Actuarial Science and Applied Statistics, Faculty of Business and Information Science, UCSI University, \\ 56000 Kuala Lumpur, Malaysia
}

Received 25 October 2021 / Accepted 13 January 2022 / Published online 15 February 2022 (C) The Author(s) 2022

\begin{abstract}
We describe the production of a high-resolution electron beam using a Penning-Malmberg buffergas trap, or Surko trap as they have become known. A high-flux beam with an energy width of $\sim 30 \mathrm{meV}$ (FWHM) is readily achieved and the efficiency of production is considerably higher than that for positrons in a similar trap configuration. The reasons for this become apparent when one considers the molecular collisions and the respective selection rules involved, for electrons and positrons. We demonstrate the production of the beam and the capacity that it realises for absolute scattering measurements and for high-resolution electron spectroscopy.
\end{abstract}

\section{Introduction and background}

The field of positron atomic physics has been revolutionised over the past few decades through the use of buffer-gas traps to produce high-resolution, variableenergy positron beams that can be used for a broad range of experiments [1]-from the production of antihydrogen [2] to low-energy atomic physics [3] and annihilation studies [4]. Such traps, in conjunction with a high-activity ${ }^{22} \mathrm{Na}$ radioactive positron source, can routinely provide a high-intensity positron beam with an energy width parallel to the guiding axial magnetic field which reflects the temperature of the trapping gases, $\left(3 / 2 \mathrm{k}_{\mathrm{B}} \mathrm{T}\right) \sim 32 \mathrm{meV}(\mathrm{FWHM})$ at room temperature.

These traps, which use high solenoidal magnetic fields $(0.5-1 \mathrm{kG})$ for radial confinement of particles and electrostatic potential for axial confinement, typically use molecular nitrogen $\left(\mathrm{N}_{2}\right)$ and carbon tetrafluoride $\left(\mathrm{CF}_{4}\right)$ as the trapping and cooling gases, with the main energy-loss mechanisms being electronic, vibrational and rotational excitation. Nitrogen is favoured for a number of reasons [5] - it has relatively low-lying electronic excited states which, when excited by positron impact, provide the principal energy loss mechanism to enable trapping of the positrons. The lowest lying of these states, the $a^{1} \Pi$ state, has an excitation threshold of $8.59 \mathrm{eV}$, which is below the threshold of $8.78 \mathrm{eV}$ for the formation of positronium (Ps) in $\mathrm{N}_{2}$ [5]. This makes it possible, to some extent, to balance the trapping of

\footnotetext{
${ }^{a}$ e-mail: Stephen.Buckman@anu.edu.au (corresponding author)
}

the inelastically scattered positrons against the potential loss of positrons through Ps formation and subsequent annihilation. It is important to note here (for the discussion that is to follow) that it is only the manifold of singlet excited states that can be excited by positron impact on the singlet $\left(X^{1} \Sigma_{g}^{+}\right)$ground state of $\mathrm{N}_{2}$. This is because the spin-orbit interaction, which can lead to spin-flip excitations, is negligible for positron collisions, and the exchange interaction, which can also lead to singlet-triplet transitions, is absent altogether for positron collisions. The principal role of the $\mathrm{CF}_{4}$ gas is to then provide further cooling of the positrons through vibrational excitations [5]. It is interesting to note that the choice of these two trapping gases, from a host of possibilities, was largely based on empirical experimental observations of trapping and cooling efficiency. The measurement of the collision cross sections for these excitation processes $[6,7]$, and thus a full understanding of the underlying molecular scattering mechanisms that enable the efficient operation of the trap [8-10], was not possible until a high-flux, high-resolution positron beam was actually realised.

Much has been written about the success of such traps for positrons and, while the trapping of electrons has also been demonstrated and explored in a number of configurations [11], to our knowledge such a trap has only been used once to produce a high-resolution electron beam for low-energy collision studies of $\mathrm{CF}_{4}[12]$.

- When one considers the various collision cross sections for electron and positron scattering from $\mathrm{N}_{2}$ and $\mathrm{CF}_{4}$, it becomes immediately obvious that the conditions for trapping and cooling electrons are far 
more favourable. This is due to a range of collisional and spectroscopic factors, namely:

The exchange interaction For electron scattering, the indistinguishability of the incident and target electrons means that electron collisions with $\mathrm{N}_{2}$ can result in the excitation of both singlet (direct excitation) and triplet (via the exchange interaction) electronic states from the singlet ground state. For positron impact, only the singlet manifold of states can be excited, due also to the absence of any significant spin-orbit interaction. In Fig. 1a (from [13]) we show the calculated potential energy curves for the singlet and triplet manifolds of $\mathrm{N}_{2}$ which identify their excited states and thresholds. In Fig. 1b, we show the corresponding electron excitation cross sections for these states [14] as well as the single ionisation cross section [15]. All of these processes lead to significant energy loss, which can then result in the trapping of the scattered or ionised electrons.

A summary of the cross sections that relate to predicting the trapping of both positrons and electrons via their energy loss when exciting the various $\mathrm{N}_{2}$ scattering channels is shown in Fig. 1c. In this case, the only measured cross sections for positron scattering are those for the $a^{1} \Pi$ state [7] and for direct ionisation [5]. These are similar to the cross sections for electrons, so we have assumed the other singlet excitations to be the same as those for electrons. The result of this comparison shows clearly that the probability of an energy-loss collision that can lead to trapping is significantly higher for electrons than for positrons.

Finally, it should be noted that the positron 'trapping' curve in Fig. 1c does not consider the losses due to positronium formation-a cross section which grows strongly above the threshold of $8.78 \mathrm{eV}\left(\mathrm{N}_{2}\right)$ to dominate the positron 'trapping' cross sections. This imposes the additional constraint of a rather narrow incident energy range of operation for the positron trap in order to maximise energy loss and trapping through electronic excitation, and minimise positron loss due to positronium formation.

- Resonant electron scattering via transient negative ions Scattering resonances, where a projectile electron is trapped temporarily in the field of the molecular target forming a transient negative ion, are ubiquitous in electron scattering and, in many (most) cases, cause significant enhancement of the cross section for the scattering channel in which they occur (see [16] for examples). To date, no such resonance effects have been clearly observed in positron scattering, although they have been observed at very low energies in positron annihilation measurements [4]. Both $\mathrm{N}_{2}$ and $\mathrm{CF}_{4}$ exhibit electron scattering cross sections which are significantly enhanced by resonance formation, particularly their vibrational excitation cross sections. In $\mathrm{N}_{2}$ for example, the ${ }^{2} \Pi_{\mathrm{g}}$ resonance in the $2-5 \mathrm{eV}$ energy range completely dominates the vibrational excitation and, without its presence, vibrational excitation of this homonuclear diatomic molecule is very small - which is the case for positron excitation. Similarly, for $\mathrm{CF}_{4}$, the excitation cross sections for the three lowest-lying vibrational modes are strongly enhanced near their thresholds, and the $\nu_{3}$ mode in particular reaches, and maintains, a significant cross section to quite high impact energies. The vibrational excitation cross sections for both $\mathrm{N}_{2}$ [17] and $\mathrm{CF}_{4}[18]$ are shown in Fig. 2a and b, respectively, and we note that these are cross section compilations based on both experiment and theory. In Fig. 2a we have summed the cross sections for the first five vibrational modes to provide a simpler overall picture, but note that the energy loss associated with these modes varies from $\sim 280 \mathrm{meV}$ for the $\nu=0-1$ mode to more than $1.3 \mathrm{eV}$ for the $0-5$ transition. Again, we emphasise here that for positrons, the effective vibrational excitation cross section for $\mathrm{N}_{2}$ will be insignificant, while those for $\mathrm{CF}_{4}$ have been shown to be similar to those measured for electrons [12], although without the sharp, near-threshold resonant enhancement that is indicated for electrons in Fig. 2b. Thus, the large, resonantly enhanced, vibrational excitation cross sections for electron- $\mathrm{N}_{2}$ scattering potentially provide a significant additional trapping and cooling mechanism for electrons over positrons.

- The birth of additional electrons through ionisation Just above the thresholds for electronic excitation lie the ionisation thresholds for both $\mathrm{N}_{2}(15.58 \mathrm{eV})$ and $\mathrm{CF}_{4}(14.7 \mathrm{eV})$. Not only does an ionising collision facilitate trapping of the incident electron through its energy loss, but a second electron is also born and this can also be trapped and cooled. Thus, it would be technically feasible, in the absence of loss mechanisms, to achieve a trapping efficiency greater than $100 \%$ for electrons through this ionisation growth channel.

- The lack of loss mechanisms for electron trapping, principally Ps formation This is an obvious difference between the electron and positron cases, but not only does it mean there are no major loss mechanisms for the electrons in the trap, it also allows for a much wider range of energies over which the trap can be tuned in the electron case in order to optimise trapping efficiency against the scattering cross sections for the two gases.

\section{Rationale for this study}

The above discussion provides a compelling background for why trapping and cooling of electrons in a Surko trap should be highly efficient. The question remains as to why one would want to do this, and what the resultant high-flux, high-resolution beam could be used for in a field where cross section measurements have been successfully conducted for a century. 


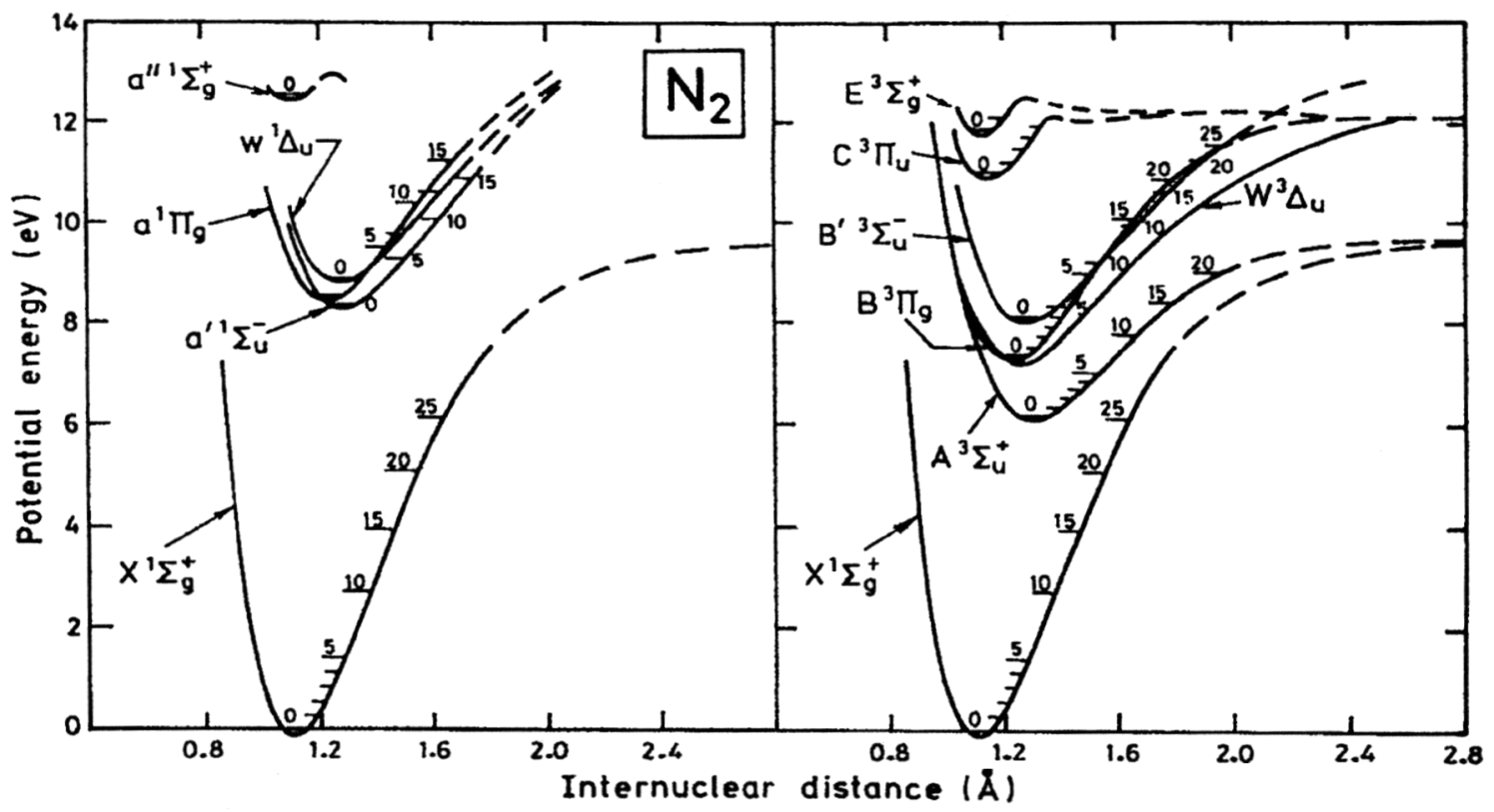

(a)

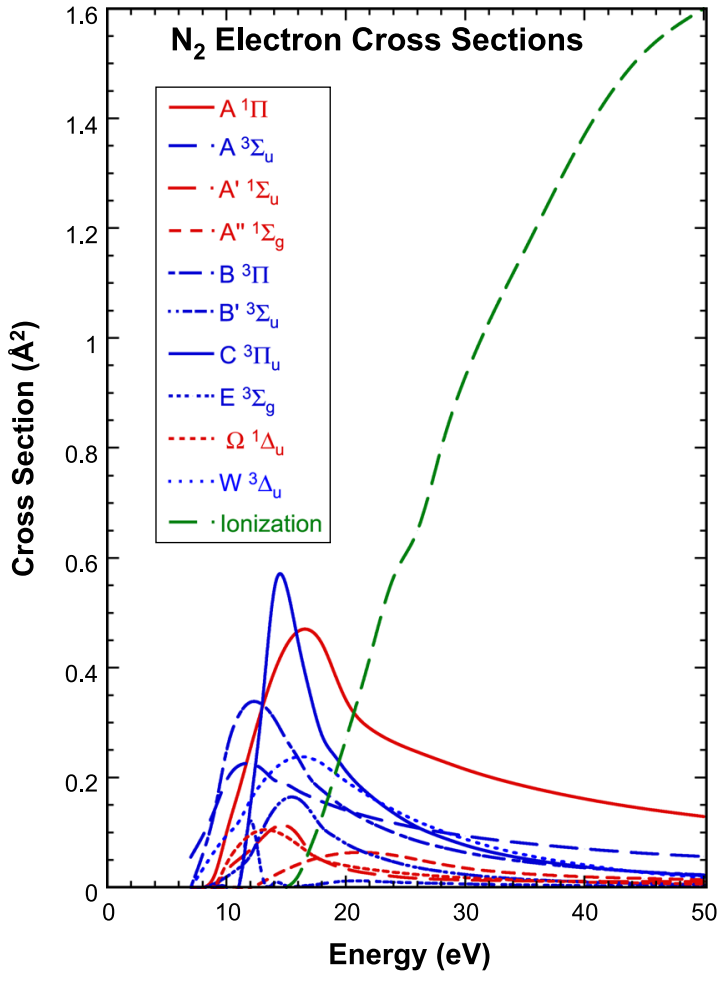

(b)

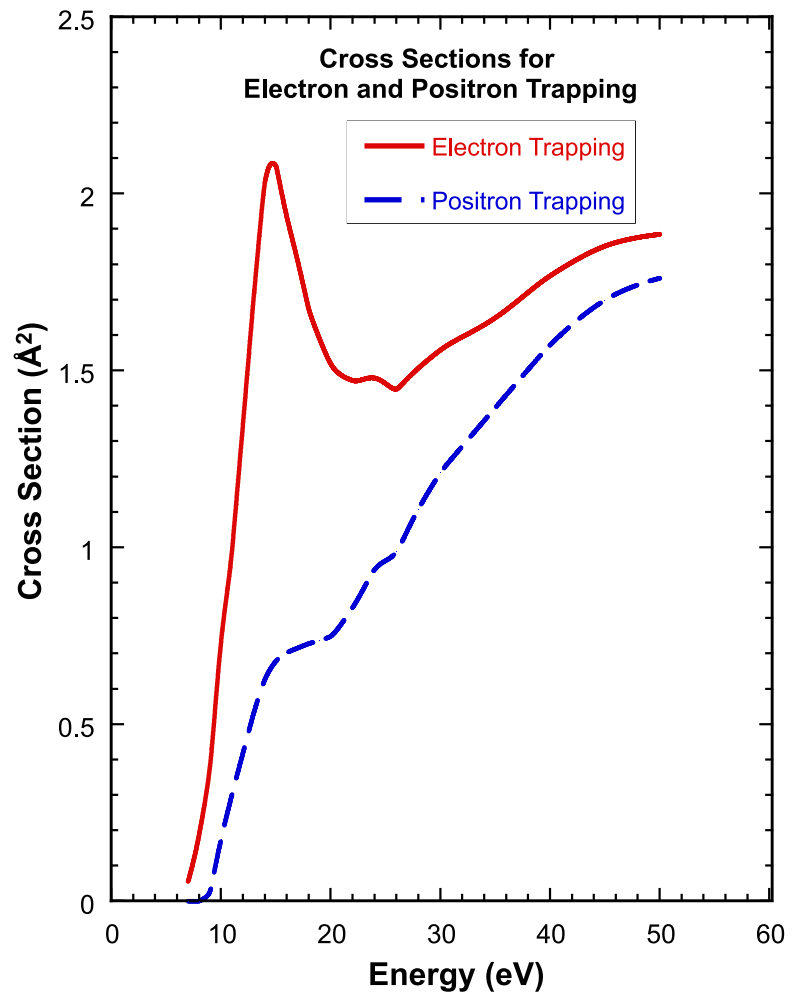

(c)

Fig. 1 a Potential energy curves for the singlet (left panel) and triplet (right panel) electronic states of $\mathrm{N}_{2}$ (from [13]). b Cross-sections for electron-impact excitation of the electronic states of $\mathrm{N}_{2}[14]$ and the single ionisation cross section [15]. Blue lines indicate singlet states (accessible by either positron or electron impact) and red lines are for the triplet states (accessible only by electron impact). c Effective trapping cross sections for electrons and positrons in $\mathrm{N}_{2}$ (see text for details) 


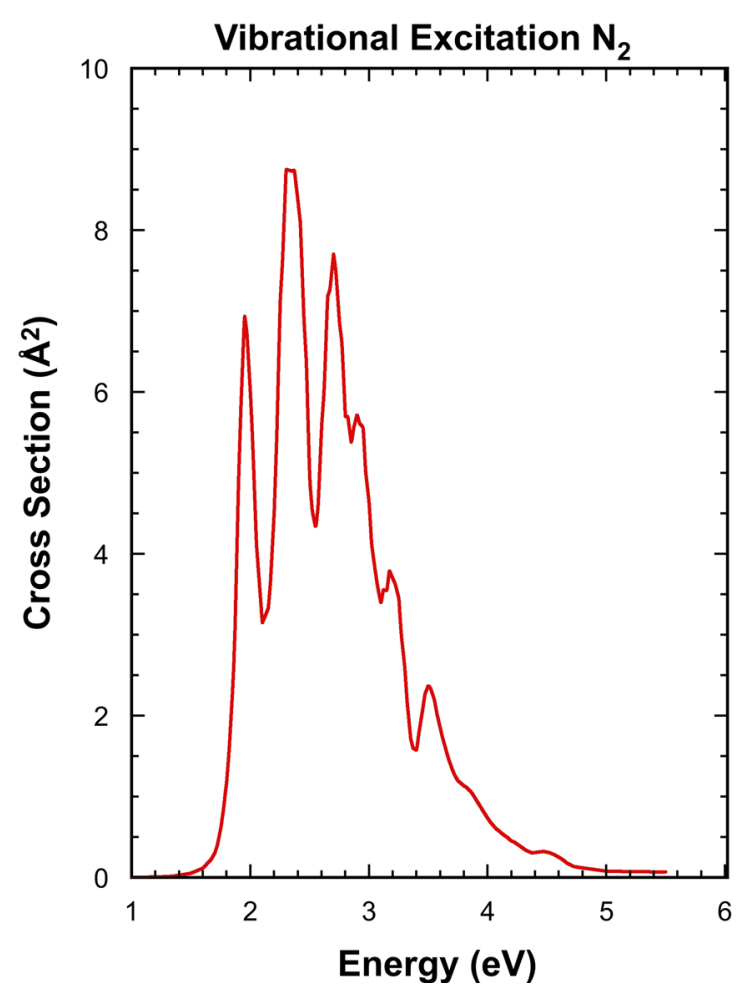

(a)

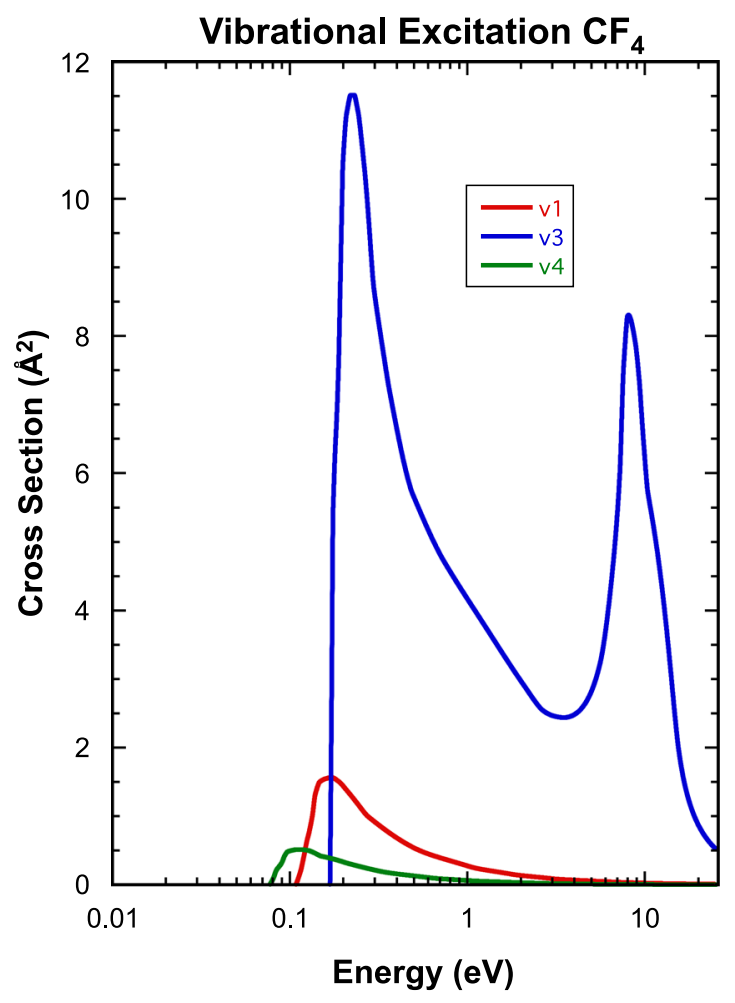

(b)

Fig. 2 a Summed cross sections for the electron impact excitation of the first five vibrational modes $(\nu=0-1,2,3,4,5)$ of $\mathrm{N}_{2}$ [17] (see text for details) and $\mathbf{b}$ Relevant vibrational excitation cross sections for electron cooling in $\mathrm{CF}_{4}$ [18] (see text for details)

Electron scattering measurements have become increasingly sophisticated over the past few decades with high precision obtained in both the measurement of scattering cross sections and electron-driven spectroscopy. One of the main drivers for this work has been the need for such cross sections in a wide range of applications - from atmospheric modelling [14, 17] to plasma processing technologies [19] and biological/medical applications [20]. For modelling these various applications, the most important and useful information that electron scattering can provide is usually at the integral or total scattering cross section level across a range of (mostly) inelastic processes, although angle-dependent measurements can also be useful, and provide a more stringent test of theory. To date, the vast majority of this integral scattering data is derived by extrapolating (to unmeasured forward and backward angles) and integrating the angular-differential measurements that are the usual outcome from highprecision electron spectrometer measurements. Without discussing the details involved, the uncertainties that arise from the integration process, together with uncertainties in the spectrometer transmission function, result in integral inelastic cross sections with significant uncertainties, usually $>20 \%$ [21].

Scattering measurements in a high magnetic field, which are possible in conjunction with a Surko trap [6], do not suffer from such effects. As has been demonstrated by various positron scattering studies [e.g. 5,
$7,12,22]$, the technique allows the direct measurement of integral cross sections for inelastic scattering, with significantly reduced uncertainties. There are a number of low-energy electron-molecule scattering systems for which discrepancies between available experimental data, and between experiment and theory for vibrational and electronic excitation, remain unresolved (e.g. $\mathrm{NO}$ [23]; $\mathrm{H}_{2} \mathrm{O}$ and some biomolecules [24, 25]; $\mathrm{CO}$ [26]; and $\mathrm{N}_{2}$ [27] to name just a few). This technique applied to electrons has the clear potential to assist in the resolution of such discrepancies. Furthermore, it would enable tests of the accuracy of phenomenological approaches, such as the BEf scaling technique [28], which has been shown to be a surprisingly useful theoretical tool for predicting electron-molecule electronic excitation cross sections.

\section{Experimental apparatus and techniques}

The multi-stage, differentially pumped beamline and Penning-Malmberg trap used for the production of the trap-based electron beam is similar to that which has been described in some detail previously in the context of positron collision measurements [29], so we shall only describe the essential features and differences here. The beamline consists of three differentially pumped stages, each evacuated by a turbomolecular pump. The 




Fig. 3 Axial potential distribution in the buffer-gas trap as a function of displacement along the trap. For reference, the trap elements are superimposed below, with element 1 on the left and 9 on the right (see text for discussion)

cylindrical stainless-steel vacuum chambers, with either $250 \mathrm{~mm}$ or $100 \mathrm{~mm}$ internal diameter, also serve as the formers for a variety of externally mounted solenoidal coils that maintain an axial magnetic field of approximately 500 Gauss along the $\sim 4 \mathrm{~m}$ of beamline.

The electron source is a simple tungsten hairpin filament, $0.1 \mathrm{~mm}$ in diameter, through which a current of approximately 2 amperes is passed to produce thermionic emission. The filament is located in a simple Pierce extraction element which can be used to regulate the electron emission. A varying negative potential can be applied to the filament to accelerate the electrons towards the first trap electrode.

The trap (Fig. 3) consists of nine gold-plated copper electrodes of varying internal diameters with the first three being $10 \mathrm{~mm}$ in diameter and 20, 60, and $60 \mathrm{~mm}$ in length, respectively. All electrodes are electrically isolated from each other and element \#8 is segmented into four quadrants to enable its operation as a 'rotating wall' electrode to compress the trapped electron beam [30]. The potential on each of the elements is also individually controlled via a 16-bit digital-toanalogue converter (NI PXI-6733), the 0-10 V output of which is coupled via a high voltage amplifier $(\times 10$ or $\times 20$ Elba Tech T-506). The RF signals for the rotating wall electrodes were produced using an arbitrary waveform generator (Rigol DG1032, $30 \mathrm{MHz}, 200 \mathrm{MS} / \mathrm{s}$ ) and a custom bias tee.

The buffer gases used for trapping and cooling are admitted to the trap in two places. $\mathrm{N}_{2}$ is admitted to the first section of the trap via a small hole in electrode 2, while $\mathrm{CF}_{4}$ is admitted at the opposite end of the trap via gaps between electrodes 4 thru 9. As discussed above, these gases provide the collisional energy loss mechanisms by which the electrons are trapped and ultimately cooled to room temperature in the final section of the trap.
Typical potential distributions for the various stages of operation of the trap (trapping, cooling, and dumping) are shown in Fig. 3. A typical $20 \mathrm{~ms}$ operating cycle of the trap involves $10 \mathrm{~ms}$ trapping time and $10 \mathrm{~ms}$ of cooling, with $\mathrm{N}_{2}$ and $\mathrm{CF}_{4}$ driving pressures of approximately 110 and 65 mTorr, respectively. It is difficult to estimate the actual pressures of the two gases in the trap but we expect that the $\mathrm{N}_{2}$ pressure in the narrow section of the trap is about 10 mTorr while that of $\mathrm{CF}_{4}$ in the last stages is about 12 mTorr, with the pumping station pressure at about $1 \mu$ Torr.

One significant difference between trapping, cooling, and transporting an electron beam in such an apparatus, as compared to a positron beam, is the issue of secondary electrons. Numerous processes downstream from the buffer-gas trap can give rise to secondary electrons-in our case the main sources are surface scattering from the collision cell apertures, and ionisation of background gas. While similar processes occur with positrons, the guiding potential fields in that case easily serve to reject any secondary electrons. This is not the case for an electron beam, where the secondary electrons are readily transported along with the trapped beam, and particular attention must be paid to both minimising/reducing these background contributions, and managing those that cannot be effectively removed. One critical component of this is the use of the 'rotating wall' electrodes to compress the beam size from around $20 \mathrm{~mm}$, or the interior diameter of the trap electrodes, to $\sim 2 \mathrm{~mm}$ as it exits the trap. The advantages of this compression are demonstrated below.

The scattering cell used for the collision measurements is constructed from non-magnetic stainless steel and is $10 \mathrm{~cm}$ in length with a $2.5 \mathrm{~cm}$ internal diameter. The entrance and exit apertures are $4 \mathrm{~mm}$ in diameter and gas is fed to the cell through Teflon tubing, which is also used for the pressure sampling line connecting the cell to an externally mounted Baratron capacitance manometer (MKS 626A.1TBF).

Energy analysis and detection of the transported beam are achieved with a simple cylindrical retarding potential analyser (RPA) and a chevron-mounted channel plate stack, which is in turn coupled to a phosphor screen. The signal from the back of the channel plates is amplified using a trans-impedance amplifier (Femto HCA-1 M-1 M-C) and the physical size of the beam can be determined by imaging the output from the phosphor with a CCD camera (Sony ICX274AL Scorpion).

The magnetic fields at the trap, collision cell, and RPA are all independently adjustable in order to facilitate inelastic scattering measurements [6], but for the present measurements they were all held at the same magnetic field of $\sim 500$ Gauss.

\section{Results and discussion}

As indicated above, the parameter space available for trapping electrons in a buffer-gas trap is considerably more favourable than for positrons. This is due both 


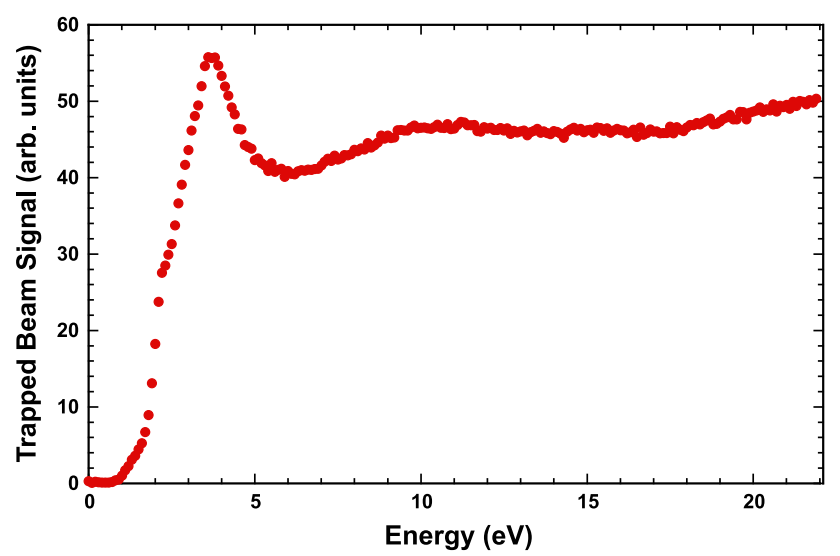

Fig. 4 Trapped beam signal obtained with the trap electrode potentials optimised for trapping on vibrational excitation in $\mathrm{N}_{2}$ and $\mathrm{CF}_{4}$ (see text for discussion)

to the lack of the Ps formation loss mechanism, which provides rather strict limits on the injection energy of positrons into such traps, and the large electron impact cross sections for vibrational excitation of $\mathrm{N}_{2}$, which are greatly enhanced by the ${ }^{2} \mathrm{P}_{\mathrm{g}}$ negative ion resonance state, but are vanishingly small for positron impact. As a result, it should be possible to trap electrons via energy loss from both electronic excitation and ionisation collisions (which are the principal trapping collisions for positrons), and also through vibrational excitation.

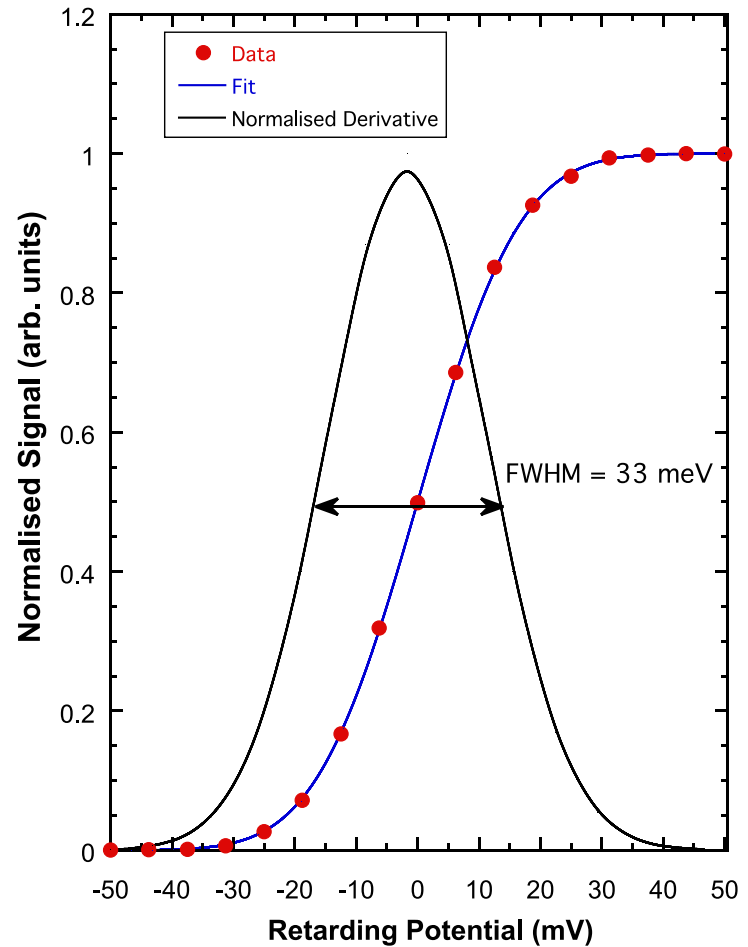

(a)
Figure 4 shows a relative trapping efficiency plot as a function of the energy of the electrons incident on the trap, noting that the energy width of this 'beam' is likely about $2 \mathrm{eV}$, when emission temperature and magnetic field differences between the emission point and trap are taken into account. At low energies, the principal trapping mechanism is energy loss through resonant vibrational excitation(s), while at higher energies energy loss through electronic excitation and ionisation will be the dominant mechanisms. As can be seen in Fig. 4, at energies below about $6 \mathrm{eV}$ there is evidence of structure which is likely explained by the convolution of the broad $( \pm 2 \mathrm{eV})$ beam entering the trap and the resonance structure present in electron impact excitation of the $\mathrm{N}_{2}$ vibrational levels (see Fig. 2a). The influence of electronic excitation and ionisation is evident at energies above $10 \mathrm{eV}$. The absolute trapping efficiency is directly proportional to the number density of the trapping gas, the energy width of the incident electron beam, the geometry of the trap, and the collision energy. While we don't have the means to directly quantify it, we estimate the absolute trapping efficiency for electrons entering the trap to be greater than $50 \%$.

In Fig. 5a we show a typical retarding field 'cut-off' curve, measured with the RPA, which gives an indication of the energy width of the pulsed, trapped beam following the magnetically guided transport through the vacuum system. This is measured by incrementally raising the potential of the analyser element to

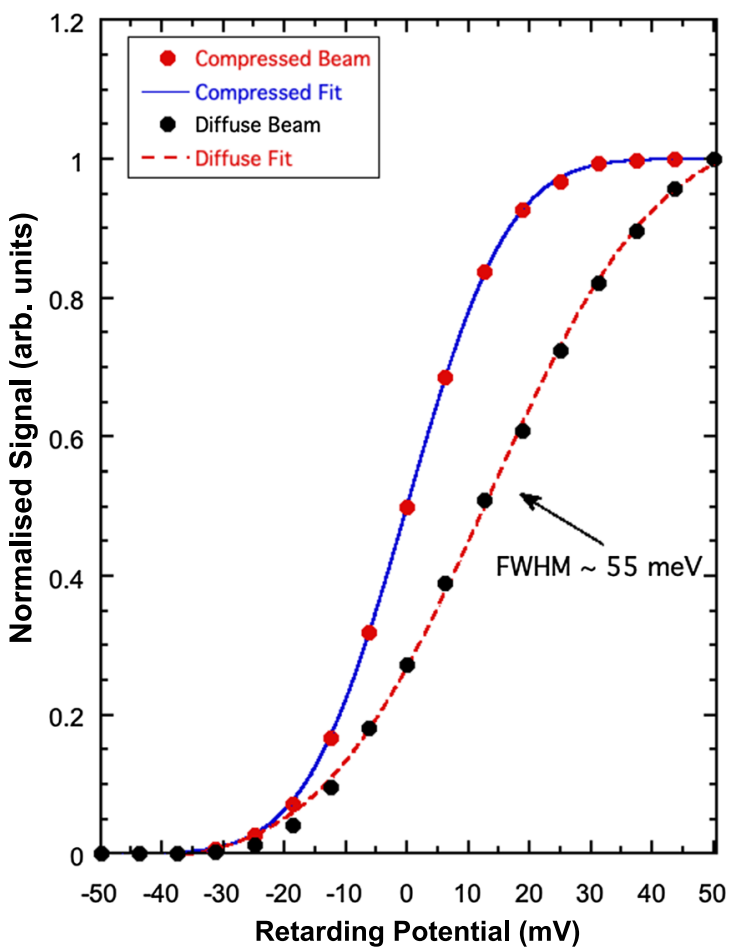

(b)

Fig. 5 a A cut-off curve demonstrating the optimum energy resolution obtained for a trapped, compressed electron beam. b A comparison of two 'cut-off' curves-one measured with the rotating wall compressing the beam and the other with a non-compressed, or diffuse, beam 


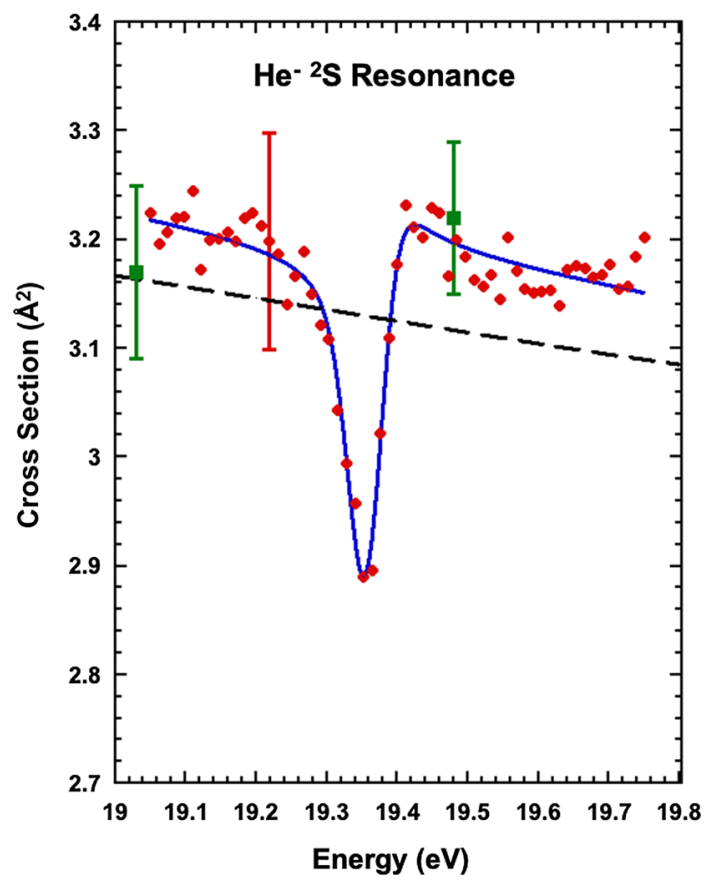

Fig. 6 The absolute elastic total cross section for helium in the region of the $\mathrm{He}^{-} 1 \mathrm{~s} 2 \mathrm{~s}^{2}{ }^{2} \mathrm{~S}_{1 / 2}$ negative ion resonance as measured with the new trap-based beam. Note that the zero is displaced. The solid line is a Fano profile fit to the experimental data (solid red circles with indicative error bar). Also shown are examples of the high-resolution, absolute measurements of Shigemura et al. [32] (solid green squares with error bars) and the variational calculation of Nesbet [33] (dashed line)

retard the transmission of the beam to the detector. In order to establish the full-width-at-half-maximum (FWHM) energy width of the beam, we have fitted the cut-off curve with an error function and then taken the derivative of this function, and this curve is also shown in Fig. 5a. The FWHM is a measure of the 'parallel' energy width of the beam and, as indicated, this is about $33 \mathrm{meV}$. In Fig. 5b we illustrate the significant advantage of using the rotating wall to compress the beam. Here we show a comparison of the energy width of the compressed beam (33 meV as in Fig. 5a) with that obtained for an uncompressed beam, in this case around $55 \mathrm{meV}$. The energy width of the uncompressed beam is broadened considerably, most likely due to the proximity of the edges of the diffuse beam to the apertures of the scattering cell where it experiences a less uniform potential distribution.

As a demonstration of the capacity of the apparatus to measure electron scattering cross sections with relatively high energy resolution, and absolute accuracy, we show a measurement of the electron-helium total elastic cross section in the region of the well-known $\mathrm{He}^{-}$ $1 \mathrm{~s} 2 \mathrm{~s}^{2} \mathrm{~S}_{1 / 2}$ negative ion resonance in Fig. 6. The experimental technique used for this measurement has been discussed in many previous works $[3,6,7]$. The most recent, and most accurate measurements of this feature are the differential elastic scattering measurements of
Gopalan et al. [31], and the total scattering measurements of Shigemura et al. [32], both of which used a high-resolution (6-8 meV), low flux electron beam produced by photo-ionisation. Gopalan et al. locate this resonance at $19.365 \mathrm{eV}$ with a width of $11.2 \mathrm{meV}$. In the total cross section measurement of Shigemura et al., the resonance appears as an asymmetric dip in the cross section with an amplitude of about $25 \%$ of the nonresonant value. The present measurement shows a similar, but broader asymmetric dip which drops about $10 \%$ below the non-resonant background cross section. The absolute cross section scale is set using the BeerLambert attenuation law through a knowledge of the scattering cell length, measurements of the scattering intensity, and the absolute pressure in the scattering cell as determined by the Baratron, which we estimate has a calibration uncertainty of about $\pm 3 \%$. This leads to an overall absolute uncertainty in the range $4-5 \%$. The agreement that we demonstrate in Fig. 6 with a sample of the previous absolute measurement, and the highly accurate variational calculations of Nesbet [33], would indicate that our overall uncertainty estimate on the absolute cross section is reasonable. Note that the Nesbet calculation is not expected to reproduce the resonance structure.

We have also fitted the resonance data in Fig. 6 with a Fano profile plus linear background, using the known resonance energy width $(11.2 \mathrm{meV})$ and energy $(19.365 \mathrm{eV})$ [31]. We obtain a shape parameter from the fit $(Q=0.205)$ which is consistent with that of Shigemura et al. $(Q=0.211)$ [32].

\section{Conclusions}

We have demonstrated the operation of a buffer-gas (Surko) trap for the production of a high resolution, high flux, pulsed electron beam that can be used for atomic and molecular collision studies. The measurement of the electron-helium total elastic cross section in the region of the well-known $\mathrm{He}^{-} 1 \mathrm{~s} 2 \mathrm{~s}^{2}{ }^{2} \mathrm{~S}_{1 / 2}$ negative ion resonance is the first observation of resonant structure using this technique and is consistent with state-of-the-art measurements.

It would be interesting to model the operation of the electron buffer-gas trap using the well-known cross sections for electron impact excitation of both $\mathrm{N}_{2}$ and $\mathrm{CF}_{4}$, as has been done extensively for positrons, with much less well-known cross sections. The key role of resonance-driven vibrational excitation in $\mathrm{N}_{2}$, the positive feedback of additional electrons produced through ionisation, and the lack of the positronium loss mechanism will undoubtedly lead to much higher efficiencies for electrons than for positrons, although the ultimate limitation on efficiency is likely to be space charge in the trap.

Acknowledgements It is an privilege to contribute this paper to the special issue in Honour of the late Professor 
Vince McKoy with whom we have shared many valuable and enjoyable interactions over the years. We gratefully acknowledge support for this research program through an Australian Research Council Discovery Grant (DP200101294); TJG is also supported in part by the U.S. National Science Foundation Award PHY-2110358. It is also a pleasure to acknowledge and thank Prof Michael Brunger and Dr Laurence Campbell from Flinders University, and Prof Zoran Petrovic of the Institute of Physics, Belgrade, who provided their data sets for $\mathrm{N}_{2}$ and $\mathrm{CF}_{4}$, respectively, and for many useful discussions. We also thank Professor Masashi Kitajima for providing the helium resonance data of Shigemura et al. in tabular form, and Professor Andrew Truscott for the use of a set of multichannel plates.

\section{Author contribution}

All authors contributed to the preparation of the manuscript. All authors have read and approved the final manuscript.

Funding Open Access funding enabled and organized by CAUL and its Member Institutions.

Data Availability Statement This manuscript has no associated data or the data will not be deposited. [Authors' comment: Data available upon request.]

Open Access This article is licensed under a Creative Commons Attribution 4.0 International License, which permits use, sharing, adaptation, distribution and reproduction in any medium or format, as long as you give appropriate credit to the original author(s) and the source, provide a link to the Creative Commons licence, and indicate if changes were made. The images or other third party material in this article are included in the article's Creative Commons licence, unless indicated otherwise in a credit line to the material. If material is not included in the article's Creative Commons licence and your intended use is not permitted by statutory regulation or exceeds the permitted use, you will need to obtain permission directly from the copyright holder. To view a copy of this licence, visit http://creativecomm ons.org/licenses/by/4.0/.

\section{References}

1. J. Fajans, C.M. Surko, Phys. Plasmas 27, 30601 (2020)

2. G.B. Andresen et al., Nature 468, 673-676 (2010)

3. A. Jones, C. Makochekanwa, P. Caradonna, D. Slaughter, J. Machacek, R.P. McEachran, J.P. Sullivan, S.J. Buckman, A.D. Stauffer, I. Bray, D. Fursa, Phys. Rev. A 83, 032701 (2011)

4. S.J. Gilbert, L.D. Barnes, J.P. Sullivan, C.M. Surko, Phys. Rev. Lett. 88, 043201 (2002)

5. J.P. Marler, C.M. Surko, Phys. Rev. A 72, 062713 (2005)
6. J.P. Sullivan, S.J. Gilbert, J.P. Marler, R.G. Greaves, S.J. Buckman, C.M. Surko, Phys. Rev. A 66(4), 042708 (2002)

7. J.P. Sullivan, J.P. Marler, S.J. Gilbert, S.J. Buckman, C.M. Surko, Phys. Rev. Lett. 87, 073201 (2001)

8. M.R. Natisin, J.R. Danielson, C.M. Surko, Phys. Plasmas 22, 033501 (2015)

9. M.R. Natisin, J.R. Danielson, C.M. Surko, J. Phys. B At. Mol. Opt. Phys. 47, 225209 (2014)

10. M.R. Natisin, J.R. Danielson, C.M. Surko, Phys. Plasmas 23, 023505 (2016)

11. C. Kurz, S.J. Gilbert, R.G. Greaves, C.M. Surko, Nucl. Inst. Meth. Phys. B 143, 188-194 (1998)

12. J.P. Marler, C.M. Surko, Phys. Rev. A 72, 062702 (2005)

13. G.J. Schulz, Rev. Mod. Phys 45, 423 (1973)

14. L. Campbell, M.J. Brunger, P.J.O. Teubner, D.C. Cartwright, JESRP 144-147, 119 (2005)

15. LXCAT https://fr.lxcat.net/cache/604aabd693eef/ ITIKAWA. J. Phys. Chem. Ref. Data 35, 31 (2006); https://doi.org/10.1063/1.1937426

16. W. Sun, M.A. Morrison, W. Isaacs, W.K. Trail, D.T. Alle, R.J. Gulley, M.J. Brennan, S. J. Buckman. Phys. Rev. A 52, 1229-1256 (1995)

17. L. Campbell, M.J. Brunger, D.C. Cartwright, P.J.O. Teubner, Planet. Space Sci. 52, 815-822 (2004)

18. M. Kurihara, Z.L.J. Petrović, T. Makabe, J. Phys. D 33, 2146-2153 (2000)

19. T. Makabe, Z. Lj, Petrovic Plasma Electronics (CRC Press, Florida, 2015)

20. B. Boudaiffa, P. Cloutier, D. Hunting, M.A. Huels, L. Sanche, Science 287, 1658 (2000)

21. M.J. Brunger, S.J. Buckman, Phys. Rep. 357, 215-458 (2002)

22. P. Caradonna, J.P. Sullivan, A. Jones, C. Makochekanwa, D. Slaughter, D.W. Mueller, S.J. Buckman, Phys. Rev. A 80, 060701 (2009)

23. M.-Y. Song, J.-S. Yoon, H. Cho et al., J. Phys. Chem. Ref. Data 48, 043104 (2019)

24. M.-Y. Song, H. Cho et al., J Phys Chem Ref Data 50, 023103 (2021)

25. M.J. Brunger, Int. Rev. Phys Chem. 36, 333 (2017)

26. M. Zawadzki, M.A. Khakoo, L. Voorneman, L. Ratkovich, Z. Maslin, K. Houfek, A. Dora, R. Laher, J. Tennyson, J. Phys. B At. Mol. Opt. Phys. 53, 165201 (2020)

27. H. Su, X. Cheng, H. Zhang, J. Tennyson, J. Phys. B 54, 115203 (2021)

28. H. Tanaka, M.J. Brunger, L. Campbell, H. Kato, M. Hoshino, A.R.P. Rau, Rev. Mod. Phys. 88, 025004 (2016)

29. J.P. Sullivan, A. Jones, P. Caradonna, C. Makochekanwa, S.J. Buckman, Rev. Sci. Inst. 79, 113105 (2008)

30. R.G. Greaves, C.M. Surko, Phys. Plas. 8, 1879-1885 (2001)

31. A. Gopalan, J. Bommels, S. Gotte, A. Landwehr, K. Franz, M.-W. Ruf, H. Hotop, K. Bartschat, Eur. Phys. J. D 22, 17-29 (2003)

32. K. Shigemura, M. Kitajima, M. Kurokawa, K. Toyoshima, T. Odagiri, A. Suga, H. Kato, M. Hoshino, H. Tanaka, K. Ito, Phys. Rev. A 89, 022709 (2014)

33. R.K. Nesbet, Phys. Rev. A 20, 58 (1979) 